\title{
Introducción al dosier temático: el Sur Global y la construcción de un nuevo Sistema Internacional
}

\author{
Lía Rodríguez de la Vega* \\ Jerónimo Delgado Caicedo* \\ Lina María Luna Beltrán ${ }^{* * *}$
}

La guerra fría fue un período determinante en la organización del Sistema Internacional. Estados Unidos, como centro del llamado 'Primer Mundo', lideró al 'mundo libre' en su confrontación con la Unión Soviética y sus aliados, también conocidos como el 'Segundo Mundo' o el 'Bloque Comunista'. Y luego, casi por descarte, estaban aquellos países en América Latina, África, Asia, el Caribe y Oceanía que no hacían parte de ninguno de los dos bloques... el llamado 'Tercer Mundo' -término acuñado por el demógrafo francés Alfred Sauvy en 1952-, los que sobraban, el resto. Y justamente esa pertenencia al grupo de los que 'no son parte' determinó, en gran medida, el funcionamiento del 'Tercer Mundo' durante décadas. Un grupo de países que ocupaban un espacio de sumisión, neocolonialismo e incluso dominación por parte de los dos bloques.

Las conferencias de Bandung, Indonesia (1955), de Belgrado, Yugoslavia (1961) y la Conferencia Tricontinental de La Habana, Cuba (1966), marcaron un punto de quiebre para el llamado 'Tercer Mundo' que se tradujo en la coordinación de esfuerzos para implementar el proyecto de un Nuevo Orden Económico Internacional (Valcárcel, 2006). Se trató de la construcción de una conciencia colectiva frente a una dinámica internacional figurada a partir de las asimetrías Norte-Sur,

\footnotetext{
* Doctora en relaciones internacionales. Secretaria General de Aladaa y miembro del Comité de Asuntos Asiáticos del Cari (Argentina). Docente-investigadora (up, unlz, Uncaus, uBA, Argentina). [liadelavega@yahoo.com]; [https://orcid.org/0000-0003-0479-1156].

* Doctor en relaciones internacionales y geografía. Docente-investigador, Facultad de Finanzas, Gobierno y Relaciones Internacionales, Universidad Externado de Colombia, Bogotá (Colombia). [jeronimo.delgado@uexternado. edu.co]; [https://orcid.org/0000-0001-9449-8281].

*** Candidata a doctora en derecho. Docente-investigadora, Facultad de Finanzas, Gobierno y Relaciones Internacionales, Universidad Externado de Colombia, Bogotá (Colombia). [lina.luna@uexternado.edu.co]; [https://orcid.org/0000-0002-8158-3724].

DOI: https://doi.org/10.18601/16577558.n34.02
} 
con un enfoque en la eliminación de las relaciones colonialistas y neocolonialistas que habían dominado el Sistema Internacional después de la segunda mitad del siglo xx.

Posteriormente, el fin de la guerra fría marcó otro hito determinante en la historia del llamado "Tercer Mundo" y los Países No Alineados, que ahora tenían que luchar por encontrar una nueva identidad. Su principal razón de existencia como concepto -una alternativa a la confrontación Este-Oeste- había desaparecido y el 'fin del Tercer Mundo', tal como lo conocíamos, se convirtió en una posibilidad (Braveboy-Wagner, 2003). Sin embargo, aun cuando el concepto mismo podría desaparecer, era evidente que los problemas que enfrentaban estos grupos de países aún existían y, en algunos casos, incluso se estaban profundizando. Así, con los cambios en el Sistema Internacional de la posguerra fría, fue necesario reconfigurar la construcción del concepto y trasladar la identidad hacia lo horizontal y cooperativista, posibilitando un nuevo diálogo Sur-Sur capaz de ofrecer un marco de acción diferente en política exterior. Esa recategorización de la realidad internacional de Asia, África y América Latina se enmarcaría dentro del concepto de Sur Global (Delgado Caicedo \& Sáenz Peñas, 2013).

Mientras algunos autores ven continuidad entre los términos "Tercer Mundo" y "Sur Global" (Grovogui, 2011), otros ven especialmente rupturas (Prashad, 2012). Escobar, por su parte, aborda los discursos asociados que aluden al Tercer Mundo en torno al impacto de un "peculiar evangelio con un fervor intenso: el desarrollo" (2007, p. 11), que formulado al inicio en Estados Unidos y
Europa, tras la segunda guerra mundial, implicaba la propuesta de "la transformación total de las culturas y formaciones sociales de tres continentes (África, América Latina y Asia), de acuerdo con los dictados de las del llamado Primer Mundo" (2007, p. 11) -confiando en su base tecnológica y económica-, punto que a su vez sería contestado.

Dados y Connell (2012), por su parte, señalan que el término Sur Global surgió alrededor del año 2000 y apuntan a que incorpora no solo espacios referidos por el término 'Tercer Mundo', sino también espacios del Norte que se caracterizan por relaciones de explotación y carácter neocolonial. Comaroff \& Comaroff (2012) concuerdan con el pensamiento abismal de Boaventura de Sousa Santos: un 'Norte' visible a este lado de la línea, frente a un 'Sur' que no existe en ninguna forma relevante $o$ comprensible al otro lado de la línea, marcados por la imposibilidad misma de existir en los dos lados al mismo tiempo (De Sousa Santos, 2007; 2009; 2010).

Sin embargo, teniendo en cuenta el papel que los países del Sur Global desarrollan en la actualidad, sería inadecuado referirse a él como un concepto negativo asociado al subdesarrollo, o a Estados fallidos, o simplemente como "el concepto geopolítico que reemplaza al 'Tercer Mundo' después del colapso de la Unión Soviética” (Traducido por los autores de Levander \& Mignolo, 2011). Por el contrario, una aproximación más positiva es necesaria y en tal sentido, Levander y Mignolo apuntan que "el 'Sur Global' es el lugar donde nuevas visiones del mundo están emergiendo y donde la sociedad global política y decolonial está teniendo lugar" (Traducido por los autores 
de Levander \& Mignolo, 2011, p. 3). Finalmente, en 2004, el Programa de las Naciones Unidas para el Desarrollo-PNUD- publicó un informe titulado 'Forging a Global South'en el cual explica no solo el concepto, sino que, además, establece qué países están ubicados en el Sur Global, apuntando que el uso del término "Sur" alude a los países en desarrollo desde la década de 1970, basándose en el hecho de que los países desarrollados e industrializados del mundo se encuentran mayormente al norte de los países en desarrollo. El término no implica aludir a la similitud de los países en desarrollo ni a su agrupación en una sola categoría, pero sí a que comparten un conjunto de vulnerabilidades y retos (Traducido por los autores de PNUD, 2004).

Aunque el tema de la distribución del poder en el Sistema Internacional ha estado en el centro de los debates académicos en relaciones internacionales por décadas, la mayoría de la literatura reciente está de acuerdo en señalar un cambio profundo en las dinámicas de poder en el sistema durante las últimas dos o tres décadas. El Indian Centre for Policy Research afirma que Estados Unidos permanece como el poder más grande, mientras China e India, en el nivel superior, continuarán creciendo. Por su parte, Brasil, Japón y Rusia podrían experimentar decrecimientos rápidos, al tiempo que Europa uno lento. También menciona la importancia de potencias medias como Corea del Sur, Indonesia, Turquía, Egipto, Irán y Sudáfrica, mientras que África y América Latina al parecer experimentarán un crecimiento rápido (Khilnani et al., 2012). Por otro lado, Cooper \& Flemes (2013) apuntan a que el surgimiento de nuevas agrupaciones de Estados del Sur global está empezando a superar la desorganización que antes silenció las contribuciones de los países emergentes en los debates de la política global.

Desde 2000, el mundo atravesó/atraviesa el cambio de poder más grande en la historia, lo cual no alude solo a los Brics sino también a otros países del Sur Global que han mejorado considerablemente su posición y liderazgo. Dentro de esta categoría se encuentran Indonesia, Sudáfrica, Turquía e Irán, conocidas por Patrick (2010) como parte de las "potencias no-occidentales principales" (p. 45); Nolte (2010) menciona a Egipto, Irán, Iraq, Israel, Nigeria, Pakistán, Arabia Saudí, Sudáfrica y Turquía; mientras que Hawksworth y Chan (2015) incluyen a Bangladesh, Colombia, Egipto, Irán, Pakistán, Filipinas, Vietnam, Nigeria, Argentina y Tailandia en el listado de países emergentes. Por su parte, Lechini y Giacaglia (2016) señalan que entre 2000 y 2015 no solo surgen nuevos poderes emergentes sino que también se dan avances que pueden observarse en materia de Cooperación Sur-Sur.

\section{POTENCIALIDADES Y DILEMAS DEL'SUR GLOBAL'}

Dirlik (2007) sostiene que los usos cambiantes del término Sur Global, y las diversas agendas implicadas, evidencian las continuidades y disparidades del posicionamiento global del "Sur" en los últimos 50 años. Agrega que los significados dados a los conceptos de "Tercer Mundo", "mundo en desarrollo", "Sur" o "Sur Global", poseen una carga simbólica que ha dependido también del contexto internacional 
e histórico, generando en este sentido expectativas distintas respecto a las potencialidades de lo que puede suceder en este escenario.

En el contexto de la Conferencia de Bandung se evidencia la conceptualización de expectativas diferentes, el Tercer Mundo como el escenario donde un "tercer camino" a la modernización podía gestarse, donde podían crearse modelos alternativos de desarrollo teniendo en cuenta las falencias de los dos sistemas y las particularidades del Sur. Un tercer mundo visto como un ente revolucionario que podía cambiar aspectos claves de la historia.

Desde finales de los 70, y posteriormente con el fin de la guerra fría, el rol del sur comenzaría a cambiar drásticamente, cuando desde el Norte e instituciones como el Banco Mundial se comienza a poner énfasis en los problemas del Sur y en la necesidad de salvarlo. Al respecto, Dirlik (2007) apunta que el Consenso de Washington es la representación última de esta nueva visión del Sur, a quien el Norte debía mostrarle un camino hacia un modelo de mercado consistente con la globalización, que se consolidaría en los 90 de manera que pudiera desarrollarse en una economía capitalista global. En línea con ello, hacia la misma época, se proyectarían, entre otros, el surgimiento de propuestas como la del Desarrollo Humano y el posdesarrollo (PNUD, 1991; Ritz, 2002), esta última con una crítica importante al discurso mismo del desarrollo.

Otro conjunto de expectativas surge en torno al concepto de los Brics, una agrupación que renueva la esperanza de caminos alternativos propuestos desde un sur que no solo parecía estar encontrando las fórmulas de crecimiento y desarrollo económico, sino que, además, comenzaba a tener una voz grupal que podía cuestionar la arquitectura institucional de la globalización (Luna, 2014). En el mundo de la academia, el debate sigue abierto; incluso en torno al tema básico de la conceptualización misma del Sur Global.

En la actualidad, con la influencia cada vez más importante de China, así como con el fortalecimiento de otros países del Sur, se hace evidente también el inicio de un nuevo escenario donde el liderazgo del Sur Global comienza a ser también un espacio de competencia. Desde este lugar es evidente también la crítica a como el Norte se ha relacionado con el Sur, sea con Estados Unidos conteniendo al Sur para que no se convierta en un reto para su hegemonía, una Unión Europea buscando mantenerse como poder normativo del Sur (Söderbaum, 2010) o la cada vez más evidente crítica al modelo de desarrollo del Norte - plasmada desde la década de 1970-, que se traduce en las diversas crisis actuales, mientras cuestiona la búsqueda desarrollista de países del Sur (Valcárcel, 2006). Todos estos temas, significados y expectativas son igualmente trascendentales para el orden global y su actual transformación, lo que hace evidente la amplitud del espectro del análisis en torno al Sur Global y su rol en el sistema internacional.

Teniendo en cuenta lo señalado, el presente dosier incluye ocho artículos que reflejan justamente esas particularidades del Sistema Internacional actual donde, sin duda, el Sur Global juega un papel cada vez más protagónico. Desde el Cuerno de África, 
pasando por China, Indonesia y Vietnam, y concluyendo con América Latina y los territorios aún colonizados del Caribe, los diferentes autores presentan textos abordados desde una variedad no solo geográfica sino también teórica que permite a los lectores de la Revista OASIS un mejor entendimiento de las dinámicas actuales en el mundo. A continuación, una breve presentación de los artículos que hacen parte de este dosier temático "El Sur Global y la construcción de un nuevo Sistema Internacional':

El primer artículo escrito por Gonzalo Ordóñez-Matamoros, María Paula Roa González y Juan Pablo Centeno Cachopo de la Universidad Externado de Colombia, Reflexiones en torno a la diplomacia cientifica: estado del debate, experiencia internacional y perspectivas para Colombia, aborda la diplomacia científica, concepto emergente en las relaciones internacionales, que considera la vinculación entre ciencia, políticas públicas y diplomacia. Apuntan a que, mientras se observa una producción dominante sobre el tema en el norte (Estados Unidos y Europa), se aprecia también una producción emergente (Brics, América Latina, África, Medio Oriente) y señalan que la diplomacia científica puede constituir una estrategia que contribuya, tanto a un mejor aprovechamiento de los recursos propios como al fortalecimiento de las capacidades científica y tecnológica de Colombia.

El segundo artículo, Percepciones sobre la politica de seguridad democrática de Colombia en la frontera Táchira-Norte de Santander (2002-2010), escrito por Ana Marleny Bustamante de la Universidad de los Andes, en Mé- rida, Venezuela, sigue las perspectivas teóricas que atienden el análisis de la seguridad de las poblaciones fronterizas y su relación con los entornos local, nacional e internacional, y busca avaluar los cambios en la percepción sobre la política de seguridad democrática de Colombia (PSD) y su efecto en la seguridad de la frontera entre Táchira y Norte de Santander (TNS) en el período citado. Su estudio cualitativo, realizado con entrevistas a informantes, resulta en la emergencia de tres categorías centrales: Ambivalencia sobre la capacidad del Estado, oscilaciones en la credibilidad del Estado y pérdida de la calidad de vida, que, consideradas integralmente en el estudio, permiten señalar que la PSD fortaleció relativamente la capacidad del Estado y fue percibida con impactos negativos en la dinámica del tNs y en la seguridad pública fronteriza.

En tercer lugar, desde la Universidad de Puerto Rico, Mayra Vélez Serrano presenta su texto Paradiplomacia en tiempos de desastre en el Caribe: un análisis de las islas nosoberanas. El artículo señala que, atendiendo a su ambigüedad política y geográfica, las islas-territorios no soberanos del Caribe han aprovechado la paradiplomacia para imprimir agencia a sus propios intereses, participando así de organizaciones regionales e internacionales y extendiendo lazos de cooperación en múltiples áreas, beneficios que se verían interpelados ante la aparición de los huracanes Irma y María, que azotaron devastadoramente la zona con semanas de diferencia en 2017. La autora se propone entender de qué modo las islas-territorios pertenecientes a Francia, Estados Unidos, Países Bajos y Reino Unido 
buscaron alternativas paradiplomáticas para responder a la emergencia planteada, al tiempo que propone como hipótesis tentativa la idea de que los marcos legales son condición necesaria, pero no suficiente para explicar la variación en la actividad paradiplomática en la zona.

En cuarto lugar, desde la Universidad de Buenos Aires, Ricardo Agustín Benítez presenta su artículo titulado Las politicas de inserción estratégica de los países del golfo Pérsico en el cuerno de África. El texto se centra en la relación de dos regiones del mundo que históricamente no han sido analizadas en conjunto y trasciende la división geopolítica tradicional de Medio Oriente y Norte de África, por un lado, y África Subsahariana por el otro. El autor también innova en su aproximación en la medida en que analiza las estrategias de inserción en el Cuerno de África utilizadas por los países más importantes del Golfo Pérsico, no como un fenómeno unidireccional, sino que también tiene en cuenta las respuestas, ya sea de cooperación o de resistencia, de países como Djibouti, Eritrea, Etiopía, Somalia, Sudán, Sudán del Sur, Kenia y Uganda.

El quinto artículo, Una comparativa de los esquemas de ciberseguridad de China y Estados Unidos, escrito por Germán Alejandro Patiño Orozco del Tecnológico de Monterrey, resalta la relevancia cada vez más importante de la ciberseguridad como herramienta del poderío nacional y la competencia internacional, apuntando la ciberseguridad del Estado en un escenario con delantera privada. En la búsqueda de responder ¿con qué fin los Estados utilizan las actividades de ciberseguridad?, considera los casos de Estados Unidos y China para analizarlos de forma comparativa, en un contexto de disputa hegemónica, resaltando que la principal diferencia entre los dos esquemas es el concepto que cada uno tiene del concepto de seguridad: China como una herramienta más política y social, y Estados Unidos con un enfoque más técnico y hegemónico.

El sexto artículo, Diplomacia económica: aproximaciones conceptuales y su aplicación en la politica de Xi Jinping hacia el Sur Global, escrito por Florencia Rubiolo (Universidad Siglo 21) y Virginia S. Busilli (Universidad Católica de Córdoba), parte del área de la economía política internacional para mostrar su evolución a lo que hoy se conoce como diplomacia económica, cuya principal característica es la utilización de instrumentos económicos para perseguir objetivos políticos. Desde allí, analizan las características de la política exterior de China y concluyen que la Iniciativa de la Franja y la Ruta tiene como principal objetivo convertir la riqueza china en poder, respondiendo tanto a intereses internos como de consolidación de su rol como poder regional, al tiempo que reafirman que con este proyecto China prioriza el Sur Global.

Desde el Centro de Investigaciones de Política Internacional (Cipi) en Cuba, Ruvislei González Sáez envía el séptimo artículo, Vietnam, el último tigre asiático, en tiempos de Covid-19: resiliencia y crecimiento, que realiza un abordaje de Vietnam, actor en ascenso con un protagonismo regional tanto desde el punto bilateral como multilateral, en la búsqueda de explicar los elementos que determinaron su relevancia en el actual contexto, pese a la compleja situación global que se vive en el marco 
de la pandemia. Dando cuenta del proceso de reforma emprendido por el país que lo sitúa como uno de los últimos tigres asiáticos, revisa el desempeño económico del país y su proyección futura, apuntando que se encuentra entre los pocos países del mundo que pudieron mantener un crecimiento del PBI gracias a su profundización de la Cooperación Sur-Sur en tiempos de pandemia.

El último artículo del dosier, escrito por Laura Daniela Molina Cortés de la Pontificia Universidad Javeriana y titulado Indonesia y su proceso de consolidación como potencia media bajo el periodo presidencial de Joko Widodo (2014-2017) (2017-2019), analiza el desempeño económico indonesio estimulado por sus diversos recursos, destacando la prioridad dada a la diplomacia multilateral y el abordaje de asuntos humanitarios bajo el gobierno de Widodo. Sostiene que Indonesia continúa consolidándose como potencia media en este período, reflejado ello en su crecimiento económico, que le permitió obtener una posición de privilegio al interior del G-20 y en la importancia asignada a los asuntos humanitarios, como se mencionara.

Para finalizar, los ocho artículos presentados en este dosier se convierten en un aporte para cuestionar el monopolio histórico que ha tenido el Norte en el conocimiento sobre relaciones internacionales. Los autores que provienen desde Colombia, Argentina, México, Venezuela, Cuba y Puerto Rico hacen un aporte importante a la construcción de conocimiento y análisis sobre el Sur Global desde el Sur Global y, particularmente, desde América Latina. Como editores invitados a este número de la revista, agradecemos a Martha
Ardila por su confianza y a los autores por sus contribuciones.

\section{REFERENCIAS}

Braveboy-Wagner, J. A. (2003). The Foreign Policy of the Global South: Rethinking Conceptual Framework. Boulder (CO), United States: Lynne Rienner Publishers.

Comaroff, J. \& Comaroff, J. L. (2012). Theory from the South: Or, how Euro- America is Evolving Toward Africa. Anthropological Forum, 22(2), 113-131.

Cooper, A. F. \& Flemes, D. (2013). Foreign Policy Strategies of Emerging Powers in a Multipolar World: an introductory review. Third World Quarterly, 34(6), 943-962. https://doi.org/10.1080/0143 6597.2013.802501

Dados, N. \& Connell, R. (2012). The Global South. Contexts 11(1), 12-13.

De Sousa Santos, B. (2007). Beyond Abyssal Thinking: From Global Lines to Ecologies of Knowledge. Review, xxx (1), 45-89.

De Sousa Santos, B. (2009). Una epistemología del sur: la reinvención del conocimiento y la emancipación social. Buenos Aires, Argentina: Consejo Latinoamericano de Ciencias Sociales - Clacso.

De Sousa Santos, B. (2010). Para descolonizar el occidente: Más allá del pensamiento abismal. Buenos Aires, Argentina: Consejo Latinoamericano de Ciencias Sociales - Clacso; Prometeo Libros.

Delgado Caicedo, J. \& Sáenz Peñas, A. (2013). Deconstruyendo el Sur Global: Una aproximación a las nuevas estrategias de la Cooperación Sur - Sur frente a los saberes hegemónicos. En L. Rodríguez de la Vega \& F. Lavolpe (Eds.), Diversidades. Asia y África en perspectiva desde América del Sur (pp. 
104-120). Lomas de Zamora, Argentina: Universidad Nacional de Lomas de Zamora.

Dirlik, A. (2007). Global South: Predicament and Promise. En The Global South, vol. 1, No. 1 (Winter 2007) (pp. 12-23). Indiana University Press.

Escobar, A. (2007). La invención del Tercer Mundo. Construcción y deconstrucción del desarrollo. Caracas: Fundación Editorial El perro y la rana.

Grovogui, S. (2003). Postcoloniality in the Global South Foreign Policy: A Perspective. En BraveboyWagner, J. A. (ed.), The foreign policy of the Global South: rethinking conceptual frameworks. Lynne Rienner Publishers, Inc.

Hawksworth, J. \& Chan, D. (2015). The World in 2050: Will the shift in global economic power continue? London, United Kingdom: PwC. Retrieved from http://www.pwc.com/gx/en/issues/the-economy/ assets/world-in-2050-february-2015.pdf

Khilnani, S.; Kumar, R.; Mehta, P. B.; Menon, P.; Nilekani, N.; Raghavan, S. and Varadarajan, S. (2012). Nonalignment 2.0: A foreign and strategic policy for India in the twenty first century. New Delhi, India: National Defence College \& Centre for Policy Research.

Levander, C. \& Mignolo, W. (2011). Introduction: The Global South and World Dis / Order. The Global South, 5(1), 1-11.

Lechini, G. and Giaccaglia, C. (Eds.) (2016). Poderes emergentes y Cooperación Sur-Sur:perspectivas desde el Sur Global. Rosario: UNR Editora.
Luna, L. (2014). La C de los Brics: El rol de China en la consolidación del Grupo. En OASIS, No. 19, enero-junio.

Nolte, D. (2010). How to compare regional powers: analytical concepts and research topics. Review of International Studies, 36(99), 881-901. https:// doi.org/10.1017/S026021051000135X

Prashad, V. (2012). The Poorer Nations: A Possible History of the Global South. London: Verso.

Patrick, S. (2010). Irresponsible Stakeholders? The Difficulty of Integrating Rising Powers. Foreign Affairs, 89(6), 44-53.

PNUD. (2004). Forging a Global South. New York City (NY), United States: United Nations Development Programme. Retrieved from http://www. undp.org/content/dam/china/docs/Publications/ UNDP-CH-PR-Publications-UNDay-for-SouthSouth-Cooperation.pdf

PNUD. (1991). Desarrollo Humano. Informe 1991. Bogotá: Tercer Mundo Editores. Recuperado de http:// hdr.undp.org/sites/default/files/hdr_1991_es_ completo_nostats.pdf

Ritz, G. (2002). El desarrollo, historia de una creencia occidental. Madrid: Universidad Complutense.

Söderbaum, F. \& Stålgren, P. (2010). The EU and the Global South. En Söderbaum, F. \& Stålgren, P. (Eds.), The European Union and the Global South, Colorado: Lynne Reinner Publishers.

Valcárcel, M. (2006). Génesis y evolución del concepto y enfoques sobre el desarrollo. Lima: PUCP-Cisepa. 